PROCEEDINGS OF THE

AMERICAN MATHEMATICAL SOCIETY

Volume 133, Number 6, Pages 1721-1722

S 0002-9939(04)07701-9

Article electronically published on December 20, 2004

\title{
REMARKS ON A RESULT OF KHALIL AND SALEH
}

\author{
T. S. S. R. K. RAO
}

(Communicated by Joseph A. Ball)

\begin{abstract}
We give a short proof of a recent result that describes onto isometries of $L(X, Y)$ for certain pairs of Banach spaces $X, Y$.
\end{abstract}

\section{Result}

Definition 1 (4). Let $X, Y$ be Banach spaces. The pair $(X, Y)$ is called an ideal pair if both are reflexive, $X^{*}$ has the approximation property, $X$ and $Y^{*}$ are strictly convex and $K(X, Y)$ is an $M$-ideal in $L(X, Y)$.

The main result of [4] describes onto isometries of $L(X, Y)$ for an ideal pair. In this short note we point out a geometric procedure for describing onto isometries of $L(X, Y)$, once a description of isometries of $K(X, Y)$ is known (Theorem 1.1 of [4]). Thus our approach yields a simpler proof of the above-described result and also enlarges the class of Banach spaces for which this result is valid.

Our idea is based on well-known results in the general structure of Banach spaces which are $M$-ideals when canonically embedded in their biduals ( $M$-embedded spaces; see [3], Chapters III and VI).

First we note that the hypothesis of reflexivity and the assumption that compacts form an $M$-ideal implies that $L(X, Y)$ is indeed the bidual of $K(X, Y)$ and the inclusion map is the canonical embedding of the space in its bidual (see [2] and [5]). Thus we are in the situation of the so-called $M$-embedded spaces.

It is to be noted that if one were to only assume that $K(X, Y)$ is an $M$-ideal in its bidual, then this hypothesis implies that $X$ is reflexive, $Y$ is an $M$-embedded space and the additional hypothesis of the approximation property leads to $L\left(X, Y^{* *}\right)$ being the bidual of $K(X, Y)$; see [5].

Thus a description of isometries of $L\left(X, Y^{* *}\right)$ in this situation involves knowing information on isometries of the bidual of $M$-embedded spaces. Let $Z$ be an $M$-embedded space. According to Proposition 2.2 of chapter III in [3], any onto isometry $T$ of $Z^{* *}$ is of the form $S^{* *}$ for an onto isometry $S$ of $Z$. This is the key ingredient of our remark (we recall the analogy with $Z=K\left(\ell^{2}\right)$ ).

Theorem 2. Let $(X, Y)$ be an ideal pair. Any onto isometry of $L(X, Y)$ is given by $T \rightarrow U T V$ for some onto isometries $U$ and $V$ of $Y$ and $X$ respectively.

Received by the editors October 11, 2003 and, in revised form, February 6, 2004.

2000 Mathematics Subject Classification. Primary 46B20.

Key words and phrases. Isometries, $M$-embedded spaces. 
Proof. By our earlier remarks any onto isometry of $L(X, Y)$ is the bitranspose of an onto isometry of $K(X, Y)$. Thus we are reduced to the study of onto isometries of $K(X, Y)$. As we now have a general way of lifting isometries of compacts, the conclusion follows from Theorem 1.1 of [4].

Remark 3. Under an appropriate assumption of the approximation property that ensures $K(X, Y)=X^{*} \otimes^{\vee} Y$, it can be seen that when $X$ is reflexive and $Y$ is an $M$-embedded space, $X$ and $Y^{*}$ are strictly convex, the arguments given during the proof of Theorem 1.1 in [4] go through giving a description of the onto isometries exactly as in Theorem 1.1. Thus when $K(X, Y)$ is an $M$-embedded space with appropriate assumptions of strict convexity and the approximation property, the isometries of the bidual, $L\left(X, Y^{* *}\right)$ have a description similar to the one given above. We also recall that by e) of Theorem III.4.6 of [3], any $M$-embedded space can be renormed so that the dual space is strictly convex and the space is still $M$-embedded.

We also take this opportunity to point out that Theorem 2.2 of 4 is a part of general folklore. To see this we note the well-known identification of $K\left(\ell^{1}\right)$ with $C\left(\beta(N), \ell^{1}\right)$ (similarly $L\left(\ell^{1}\right)$ can be identified with the $\ell^{\infty}$-direct sum of $\ell^{1}$ ) and the general Banach-Stone theorem that describes onto isometries of these spaces ([1]).

Note added on 1-9-04. More information on isometries is available in my articles: 1) Some generalizations of Kadison's theorem: A survey, Extracta Mathematicae 19 (2004) and 2) Isometries of spaces of operators, preprint. It is shown here that Theorem 2.1 of [4] is incorrect and the assumption of strict convexity cannot be dropped on $Y^{*}$ in Theorem 1.1 of [4].

\section{REFERENCES}

[1] E. Behrends, M-structure and the Banach-Stone theorem, Springer LNM No. 736, Springer, Berlin, 1979. MR0547509 (81b:46002)

[2] C. M. Cho, Spaces of compact operators which are $M$-ideals in $\mathcal{L}(X, Y)$, Internat. J. Math. and Math. Sci. 15 (1992) 617-620. MR1169829 (93h:47054)

[3] P. Harmand, D. Werner and W. Werner, M-ideals in Banach spaces and Banach algebras, Springer LNM No. 1547, Springer, Berlin 1993. MR1238713 (94k:46022)

[4] R. Khalil and A. Saleh, Isometries of certain operator spaces, Proc. Amer. math. Soc., 132 (2004) 1483-1493. MR.2053355

[5] T. S. S. R. K. Rao, Space of compact operators as an M-ideal in its bidual, Extracta Mathematicae 7 (1993) 114-118. MR.1248457 (94k:46037)

Stat-Math Unit, Indian Statistical Institute, R. V. College P.O., Bangalore 560059, INDIA

E-mail address: tss@isibang.ac.in 